ACta Univ. Sapientiae, Informatica 11, 1 (2019) 24-40

DOI: $10.2478 /$ ausi-2019-0002

\title{
A study on the pendant number of graph products
}

\author{
Jomon K. SEBASTIAN \\ Manonmaniam Sundaranar University \\ Tirunelveli, Tamil Nadu 627012, INDIA \\ email: jomoncmi@gmail.com \\ Sudev NADUVATH \\ CHRIST (Deemed to be University) \\ Bangalore, India \\ email: sudev.nk@christuniversity.in
}

\author{
Joseph Varghese \\ KUREETHARA \\ CHRIST (Deemed to be University) \\ Bangalore, India \\ email: frjoseph@christuniversity.in \\ Charles DOMINIC \\ CHRIST (Deemed to be University) \\ Bangalore, India \\ email: \\ charles.dominic@christuniversity.in
}

\begin{abstract}
A path decomposition of a graph is a collection of its edge disjoint paths whose union is $G$. The pendant number $\Pi_{p}$ is the minimum number of end vertices of paths in a path decomposition of G. In this paper, we determine the pendant number of corona products and rooted products of paths and cycles and obtain some bounds for the pendant number for some specific derived graphs. Further, for any natural number $\mathrm{n}$, the existence of a connected graph with pendant number $\mathrm{n}$ has also been established.
\end{abstract}

\section{Introduction}

We refer to West [1] and Harary[2] for terms and definitions in graph theory. All graphs we consider in this paper are undirected, simple, finite and connected.

Computing Classification System 1998: G.2.2

Mathematics Subject Classification 2010: 05C70, 05C76, 05C38

Key words and phrases: graph operations, path decomposition, pendant number 
Partition of a graph $\mathrm{G}$ into its subgraphs is also termed as decomposition of G. A path-decomposition of a graph $\mathrm{G}$ is the partitioning of its edges into subgraphs $S_{i}, 1 \leq i \leq n$, where each of the subgraph $S_{i}$ is a path in $G$.

Definition 1 [3] The pendant number of a graph $\mathrm{G}$, denoted by $\Pi_{p}(\mathrm{G})$, is the least number of vertices in a graph such that they are the end vertices of a path in a given path decomposition of a graph $G$. If $V_{p}(G)$ denotes the set of all $u \in V(G)$ such that $u$ is an end vertex of a path in P-decomposition in $G$, then $\Pi_{p}(G)=\min \left\{\left|V_{p}(G)\right|\right\}$.

An introductory study on pendant number of graphs is available in [3]. A similar study on the star number of graphs can be seen in [4]. For the discussions in this paper, we use the following theorems.

Theorem 2 [3] Let $\mathrm{G}$ be a connected graph with $\mathrm{n}$ vertices. If $\mathrm{G}$ has $\mathrm{l}$ odd degree vertices, then $\mathrm{l} \leq \Pi_{\mathrm{p}}(\mathrm{G}) \leq \mathrm{n}$.

Theorem 3 [3] Let $\mathrm{T}$ be tree on $\mathrm{n}$ vertices of which $\mathrm{k}$ vertices are of even degree. Then, $\Pi_{\mathrm{p}}(\mathrm{T})=\mathrm{n}-\mathrm{k}$.

Theorem 4 [3] For a unicyclic graph $\mathrm{G}$ of order $\mathrm{n} ; \mathrm{n} \geq 3$ with $\mathrm{l}$ odd degree vertices, we have

$$
\Pi_{p}(G)= \begin{cases}2 & \text { if } m=0 \\ l+1 & \text { if } m=1 \\ l & \text { otherwise }\end{cases}
$$

where $\mathrm{m}$ is the number of vertices on $\mathrm{C}$ with $\operatorname{deg}(v) \geq 2$.

Proposition 5 [3] If $\mathrm{G}$ is the cycle $\mathrm{C}_{\mathrm{n}}$ on $\mathrm{n} \geq 3$ vertices, then $\Pi_{\mathrm{p}}(\mathrm{G})=2$.

\section{Properties of pendant number}

Even though there is no direct relation between the pendant number and other known and popular graph parameters, it is observed that the pendant number is highly influenced by the number of odd degree vertices in a graph. Moreover, the pendant number of a graph $G$ has plenty of interesting properties, some of which, we deal with in the following discussion.

Definition 6 A $\Pi_{p}$-realisation of a positive integer $k \geq 2$ is a minimal connected graph $\mathrm{G}$, whose pendant number is $k$. 
By the one-point union of a collection of graphs (possibly with different order), we mean a graph obtained by replacing some or all edges of a path $\mathrm{P}$ by some graphs in the collection. In view of this notion, the following theorem establishes the existence of $\Pi_{p}$-realisation for any given positive integer.

Theorem 7 For every positive integer $k \geq 2$, there exists a $\Pi_{p}$-realisation for k.

Proof. We can iteratively construct a (minimal) connected graph with pendant number $k$ using 1 -point union of $K_{2}$ and $K_{3}$ (in alternative manner) as shown in Figure 1. We note that for an even integer $k$, taking the one-point union of $\frac{k}{2}$ number of $\mathrm{K}_{2}$ 's and $\frac{k}{2}-1$ number of $\mathrm{K}_{3}$ 's alternatively, we can construct a graph with $\Pi_{\mathfrak{p}}(\mathrm{G})=\mathrm{k}$, whereas for an odd integer $k$, taking the one-point union of $\frac{\mathrm{k}-1}{2}$ number of $\mathrm{K}_{2}$ 's and $\mathrm{K}_{3}$ 's alternatively, we can construct a graph with $\Pi_{p}(G)=k$. This completes the proof.

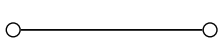

(a) A graph with $\Pi_{p}=2$.

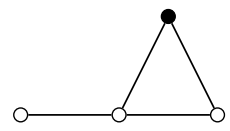

(b) A graph with $\Pi_{p}=3$.

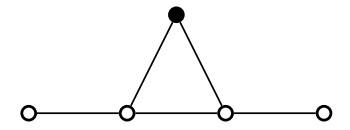

(c) A graph with $\Pi_{p}=4$.

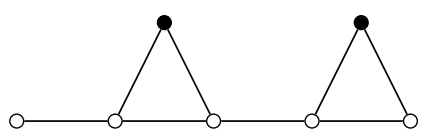

(d) A graph with $\Pi_{p}=5$.

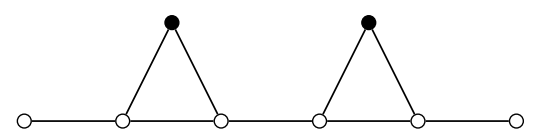

(e) A graph with $\Pi_{p}=6$.

Figure 1: $\mathrm{A} \Pi_{p}$ realisation of the given positive integer $k \geq 2$.

Remark 8 It has been determined that for every $k \in \mathbb{N}$, there exists a graph with $\Pi_{p}(G)=k$. More precisely, for every $k \in \mathbb{N}$, there exists a graph with cycles such that $\Pi_{p}(G)=k$ and there exists an acyclic graph corresponding to any even natural number.

Theorem 9 If $\mathrm{u}, \boldsymbol{v}$ are two non-adjacent vertices in a graph $\mathrm{G}$ of order $\mathrm{n}$, then $\left|\Pi_{\mathrm{p}}(\mathrm{G}+\mathrm{uv})-\Pi_{\mathrm{p}}(\mathrm{G})\right| \leq 2$.

Proof. Let $\mathrm{G}$ be a graph with $\Pi_{\mathrm{p}}(\mathrm{G})>2$. Let $u$ and $v$ be two non-adjacent vertices in $\mathrm{G}$. Here, one may consider the following two cases: 
Case-1: If $u$ and $v$ are of even degree and neither of them is an end point of a path in the decomposition of $\mathrm{G}$, then in $\mathrm{G}+\boldsymbol{u} v, u$ and $v$ are of odd degree and become end points of some path in $G$, while the degrees of all other vertices remain the same as those in G. Hence, in this case, $\Pi_{p}(G+u v)=\Pi_{p}(G)+2$ (see Figure 2 for illustration).

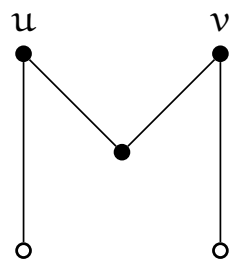

(a) A graph $G$ with no even degree vertex as end point of a path.

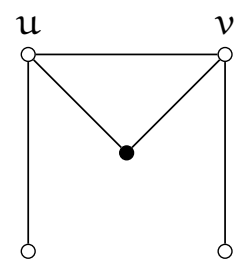

(b) A graph $\mathrm{G}+u \mathfrak{v}$ with $\Pi_{p}(\mathrm{G}+\mathrm{uv})=$ $\Pi_{p}(G)+2$.

Figure 2

Case-2: Let $P$ and $P^{\prime}$ be two (vertex-) disjoint paths in $G$ such that $u$ is an end vertex of $P$ in $G$ and $v$ is an end vertex of $P^{\prime}$ in $G$. Join the edge $u v$. Now the path $\mathrm{P}+\mathfrak{u} v+\mathrm{P}^{\prime}$ becomes a longer path, in which neither $u$ nor $v$ is an end point. In this case, $\Pi_{p}(G+u v)=\Pi_{p}(G)-2$ (see Figure 3 for illustration).

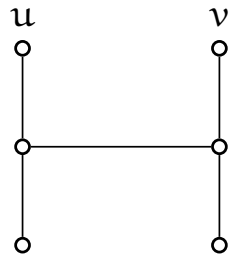

(a) A graph $G$ with end vertices of paths.

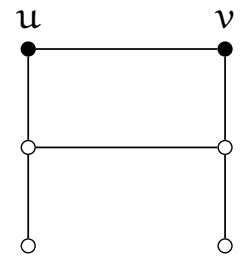

(b) A graph $G+u v$ with $\Pi_{p}(G+u v)=$ $\Pi_{p}(G)-2$.

Figure 3

It can be verified that in all other possible cases, $\Pi_{p}(G+u v)$ lies between $\Pi_{p}(G)-2$ and $\Pi_{p}(G)+2$. This completes the proof.

Invoking the results mentioned, we discuss some immediate observations in this section. By one-point union of cycles, we mean a graph obtained from a path by replacing its edges by cycles (possibly of different order). The following result provides the pendant number of one-point union of cycles. 
Proposition 10 If $\mathrm{G}$ is the one-point union of cycles, then $\Pi_{\mathrm{p}}(\mathrm{G})=2$.

Proof. The proof is clear from Figure 4. The two edge disjoint $(u, v)$-paths of the one-point union of cycles are illustrated in the figure.

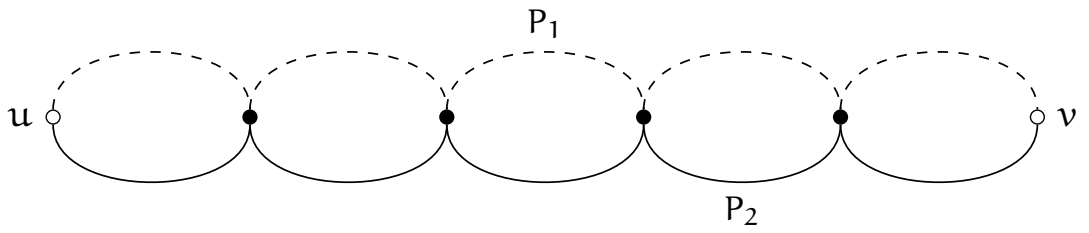

Figure 4: One-point union of cycles.

Proposition 11 Let $\mathrm{G}$ be a graph, which is neither a cycle nor a one-point union of cycles. Then, the one-point union of $\mathrm{G}$ and a cycle $\mathrm{C}_{n}$ has the pendant number $\Pi_{p}(\mathrm{G})+1$.

Proof. Let $\mathrm{G}^{*}$ be the one-point union of a given graph $\mathrm{G}$ and a cycle $\mathrm{C}_{n}$ and let $v$ be the vertex common to $G$ and $C_{n}$ in $G^{*}$. If $v$ is a pendant vertex of any path decomposition of $\mathrm{G}$, it will be a pendant vertex of some paths in $\mathrm{G}^{*}$ also. It can be taken as an end vertex of a path in $C_{n}$ too. The other end vertex of this path can be arbitrarily chosen on $C_{n}$. If $v$ is not a pendant vertex of any path decomposition of $\mathrm{G}$, some paths passing through $v$ in $\mathrm{G}$ can be extended to a vertex $u$ of $C_{n}$ in $G^{*}$. Hence, in this case also, one vertex of $C_{n}$ will be a pendant vertex in $\mathrm{G}^{*}$, other than the pendant vertices in $\mathrm{G}$.

Therefore, in both cases, the pendant number is $\Pi_{p}(G)+1$ (see Figure 5).

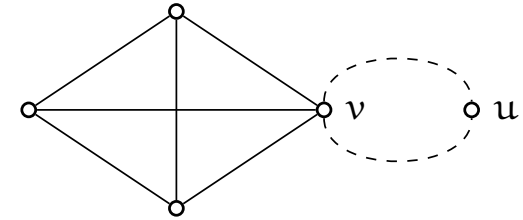

(a) The one-point union of $\mathrm{G}$ and $\mathrm{C}_{n}$ with $v$ as an end vertex of paths.

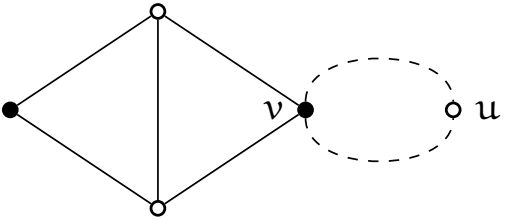

(b) The one-point union of $\mathrm{G}$ and $\mathrm{C}_{\mathrm{n}}$ with $v$ not as an end vertex of paths.

Figure 5 
Proposition 12 If $\mathrm{G}$ is a graph with cycles such that $v$ is an end vertex of a path and $\mathrm{G}^{*}$ is the one-point union of $\mathrm{G}$ and a path $\mathrm{P}_{\mathrm{m}}$ joined at $v$, then $\Pi_{p}(G) \leq \Pi_{p}\left(G^{*}\right) \leq \Pi_{p}(G)+1$.

Proof. If $v$ is an end vertex of a single path, then the path can be extended to $P_{m}$ so that $u \in P_{m}$ is the end vertex of the resultant path (see Figure 6a). If $v$ is the end vertex of more than one path, then $v$ together with $u \in P_{m}$ become the end vertices of the new path (see Figure $6 \mathrm{~b}$ ).

Example 13 Let $G=K_{n} ; n$ even and $G^{*}$ be the ( $\left.n, m\right)$-shovel graph, which is the one-point union of $K_{n}$ and $P_{m}$ (that is, $\left.\left(K_{n} \cup \dot{P} P_{m}\right)\right)$ [7]. Then, $\Pi_{p}\left(G^{*}\right)=$ $\Pi_{p}(G)$. Let $G=K_{n} ; n$ odd and $G^{*}$ be the $(n, m)$-shovel graph. Then, $\Pi_{p}\left(G^{*}\right)=$ $\Pi_{p}(G)+1$.

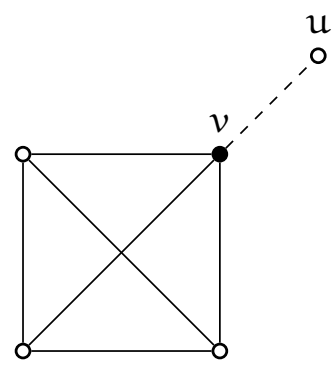

(a) A graph $G$ with $\Pi_{p}\left(G^{*}\right)=\Pi_{p}(G)$

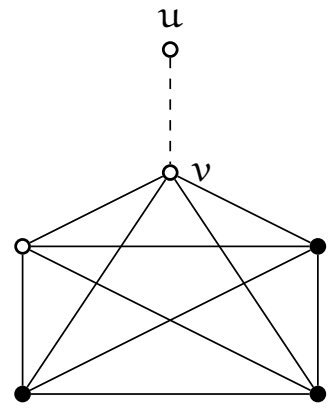

(b) A graph $G$ with $\Pi_{p}\left(G^{*}\right)=\Pi_{p}(G)+1$.

Figure 6

A maximal, bi-connected, edge-disjoint subgraph of a graph $\mathrm{G}$ is called a block of $\mathrm{G}$. Note that two blocks may have utmost one vertex in common.

Proposition 14 If $\mathrm{B}_{1}, \mathrm{~B}_{2}, \ldots, \mathrm{B}_{\mathrm{k}}$ are $\mathrm{k}$ distinct blocks of a graph $\mathrm{G}$, then $\Pi_{p}(\mathrm{G}) \leq \sum_{i=1}^{k} \Pi_{p}\left(B_{i}\right)$.

The Figure 4 explains the case when the equality in the above result holds. The next proposition establishes an illustration of the case where there is the strict inequality in the above proposition.

Definition 15 A non-uniform friendship graph $\mathrm{F}_{\mathrm{n}}^{*}$ is defined as the graph obtained by joining $n$ cycles (need not be of same order) to a common vertex. Cycles in this graph are called as petals of $\mathrm{F}_{n}^{*}$. 
The following result discusses the pendant number of non-uniform friendship graphs.

Proposition 16 For a non-uniform friendship graph $\mathrm{F}_{\mathfrak{n}}^{*}$ on $\mathrm{n}$ petals, $\Pi_{\mathfrak{p}}\left(\mathrm{F}_{\mathfrak{n}}^{*}\right)=$ n.

Proof. Let there be $n$ petals in $F_{n}^{*}$. Let $v_{0}$ be the common vertex and choose $n$ vertices $v_{1}, v_{2}, \ldots, v_{n}$ randomly from each petal. Join the path from $v_{1}$ to $v_{2}$ through $v_{0}$. Then, $v_{1}$ and $v_{2}$ becomes the end vertices of a path. Similarly, construct the paths from $v_{2}$ to $v_{3}$, from $v_{3}$ to $v_{4}, \ldots$, from $v_{n-1}$ to $v_{n}$, and from $v_{n}$ to $v_{1}$. There are $n$ such paths with end vertices of every path is a starting point of another path. Hence, $\Pi_{p}(G)=\mathfrak{n}$.

This class of non-uniform friendship graphs is another example for $\Pi_{p^{-}}$ realisation of positive integers. This fact is immediate from the above result. We consider $\delta(G)$ as the lowest degree among all the degrees of the vertices of the graph $\mathrm{G}$.

Theorem 17 There exists a connected graph $\mathrm{G}$ with $\delta(\mathrm{G}) \geq 2$ corresponding to any natural number $\mathrm{k} \geq 2$.

Proof. A (minimal) connected graph $G$ with $\delta(G) \geq 2$ can be iteratively constructed corresponding to any natural number $k \geq 2$. Consider a cycle $C_{k}$ with chords and a cycle $C_{3}$ in alternative manner. For an even integer $2 k$, take the cycle $C_{2 k}$ with every vertex has exactly one chord results in an even number as pendant number. For an odd integer $2 k+1$, take the one point union of the cycle $C_{2 k}$ with every vertex have exactly one chord and a $C_{3}$ attached to any one of its vertices, result in an odd number as pendant number (by Theorem 11). That is, consider the cycle $C_{3}$ to get the pendant number 2 (see Figure 7a). The one-point union of a diamond with $\mathrm{C}_{3}$ has the pendant number 3 (see Figure $7 \mathrm{~b}$ ). The cycle $\mathrm{C}_{4}$ with every vertex having exactly one chord has the pendant number 4 (see Figure $7 \mathrm{c}$ ). The one point union of the above $\mathrm{C}_{4}$ and a $C_{3}$ has the pendant number 5 (see Figure $7 \mathrm{~d}$ ). The cycle $C_{6}$ with every vertex having exactly one chord has the pendant number 6 (see Figure $7 \mathrm{e}$ ). The one point union of the above $\mathrm{C}_{6}$ and a $\mathrm{C}_{3}$ has the pendant number 7 (see Figure 7f). This process gives the pendant number of a (minimal) connected graph with $\delta(G) \geq 2$ for any natural number. This completes the proof.

In view of the Theorem 17 , instead of taking $C_{3}$, if one takes a cycle of desired length, then it leads to the existence of a graph with desired pendant number and desired number of vertices. Note that to get the required pendant 


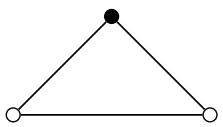

(a) A graph with $\kappa \geq 2$ (b) A graph with $\kappa \geq 2$ and $\Pi_{p}=2$.

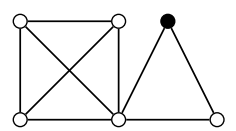

(d) A graph with $\kappa \geq$ 2 and $\Pi_{p}=5$.

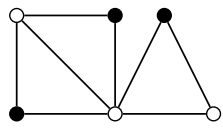
and $\Pi_{p}=3$.

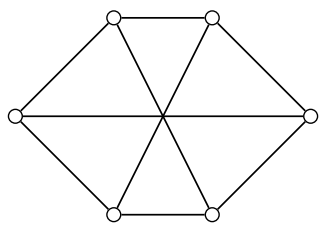

(e) A graph with $\mathrm{k} \geq 2$ and $\Pi_{p}=6$.

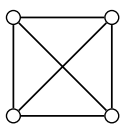

(c) A graph with $\kappa \geq 2$ and $\Pi_{p}=4$.

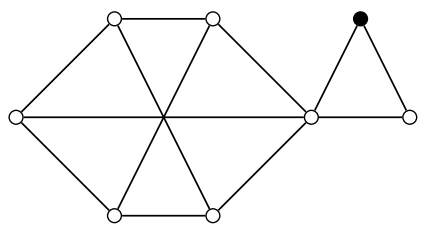

(f) A graph with $\mathrm{k} \geq 2$ and $\Pi_{p}=7$.

Figure 7

number, one must have at least the same number of odd vertices. It leads to the following result.

Corollary 18 There exists a connected graph $\mathrm{G}$ of order $\mathrm{n}$ with $\delta(\mathrm{G}) \geq 2$ corresponding to any natural number $\mathrm{k} \geq 2$ and corresponding to any order $\mathrm{n} \geq 3$ with desired pendant number.

Theorem 19 A graph $\mathrm{G}$ of order $\mathrm{n} \geq 3$, with at least one even degree vertex has the pendant number at most $\mathrm{n}-1$.

Proof. Since the result has already been proved for acyclic graphs (see Theorem 3), it is sufficient to prove the result for cyclic graphs on $n$ vertices using the method of induction on the number of vertices.

The smallest graph with cycles with at least one even degree vertex is $C_{3}$ and $\Pi_{p}\left(C_{3}\right)=2$. Hence the result is true for $n=3$. Assume the result is true for $n=k$. Let $G$ be a graph with $k$ vertices and $\Pi_{p}(G) \leq k-1$. Let $v_{1}$ be an even degree vertex, which is not an end vertex of any path in $G$.

Now let $\mathrm{n}=\mathrm{k}+1$. Add one more vertex $v_{\mathrm{k}+1}$ to the above graph $\mathrm{G}$. Connect any number of vertices of $\mathrm{G}$ except $v_{1}$ to $v_{\mathrm{k}+1}$ (if $v_{1}$ is joined to $v_{\mathrm{k}+1}$, then degree of $v_{1}$ becomes odd). For $v_{1}$ is not an end vertex of any path in G, $\Pi_{p}(G) \leq k$. Hence, the proof.

Theorem 20 Let $\mathrm{G}$ be a graph of order $\mathrm{n}$, consisting of cycles and $\mathrm{k}>0$ even degree vertices. Then, $\mathrm{n}-\mathrm{k} \leq \Pi_{\mathrm{p}}(\mathrm{G})$. 
Proof. Let us use the induction on $k$, viz., $k=1, n-1$ and $n$. Let $k=1$. Then, by Theorem 19, $n-1 \leq \Pi_{p}(G) \leq n-1$. Let $k=n-1$ then, $n-(n-1)=$ $1 \leq \Pi_{p}(G)$. Let $k=n$ then, $n-n=0 \leq \Pi_{p}(G)$. Hence the result is true.

Combining Theorem 19 and Theorem 20, it follows:

Theorem 21 If a graph $\mathrm{G}$, with $\mathrm{n} \geq 3$ vertices, has $\mathrm{k}$ even degree vertices, then $\mathrm{n}-\mathrm{k} \leq \Pi_{\mathrm{p}}(\mathrm{G}) \leq \mathrm{n}-1$.

Proposition 22 If both $\mathrm{G}$ and its complement $\overline{\mathrm{G}}$ are connected graphs with odd degree, then $|\mathrm{V}(\mathrm{G})| \geq 5$.

Proof. Let a graph $G$ and its complement $\bar{G}$ be connected graphs such that all their vertices are of odd degree. Then, at least two vertices each of $G$ and $\bar{G}$ will be with degree $\geq 3$. If all vertices except one is of degree one in $G$, then this vertex must be isolated in $\bar{G}$. It implies that the number of vertices of $G$ must be at least 5 .

A pineapple graph [9], denoted by $\mathrm{K}_{\mathrm{n}}^{\mathrm{m}}$, is a graph obtained by appending $\mathrm{m}$ pendant edges to a vertex of a complete graph $\mathrm{K}_{\mathrm{n}} ; \mathrm{m} \geq 1, \mathrm{n} \geq 3$. Let $\mathcal{A}$ be the collection of graphs given in Figure 8 and Figure 9. Even though $\mathrm{K}_{3}^{\mathrm{m}} \subseteq$ one-point union of a triangle and an odd degree tree, $\overline{\mathrm{K}}_{3}^{\mathrm{m}}$ is disconnected. Moreover, it is clear that the complement of one-point union of triangle and an odd degree tree always has $\Pi_{p} \leq n-2$ (see Theorem 3 and Theorem 4).

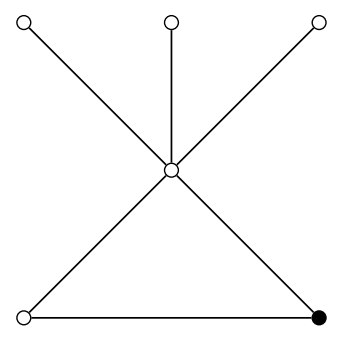

Figure 8: The graph $\mathrm{K}_{3}^{\mathrm{m}} ; \mathrm{m}$ odd.

The next proposition can be proved in a similar manner as that of Theorem 19.

Proposition 23 Let $\mathrm{G}$ be a graph $\mathrm{G}$ of order $\mathrm{n} \geq 4$ and $\mathrm{G} \notin \mathcal{A}$. If $\mathrm{G}$ has at least two even degree vertices, then $\Pi_{p}(G) \leq n-2$.

Using Proposition 22 and Proposition 23, we have the next result associated a graph $\mathrm{G}$ and its complement $\overline{\mathrm{G}}$. 


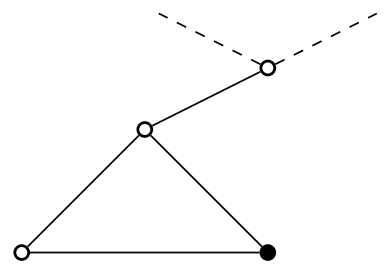

Figure 9: One-point union of $K_{3}$ and an odd degree tree.

Theorem 24 If both the graphs $\mathrm{G}$ and its complement $\overline{\mathrm{G}}$ are connected, then $4 \leq \Pi_{p}(G)+\Pi_{p}(\bar{G}) \leq 2(n-1)$.

Proof. The lower bound of $\Pi_{p}(G)$ for any graph $G$ is 2 . Therefore, $2 \leq \Pi_{p}(G)$ and $2 \leq \Pi_{p}(\bar{G})$. Hence, $4 \leq \Pi_{p}(G)+\Pi_{p}(\bar{G})$.

To prove the other part, one may consider the following two cases:

Case-1: The number of vertices $n$ of the graph $G$ is odd. Since $n$ is odd, at least one vertex each of $G$ and $\bar{G}$ must be even. Thus, the result can be determined by using the Theorem 19 , as $\Pi_{p}(G) \leq n-1$ and $\Pi_{p}(\bar{G}) \leq n-1$.

Case-2: The number of vertices $n$ of the graph $G$ is even. Let all the vertices of $G$ be odd degree vertices. Hence, $\Pi_{p}(G)=n$. Thus, the degree of $\bar{G}$ must be even. By Proposition 22, $|\mathrm{V}(\mathrm{G})| \geq 5$ and by Proposition 23, $\Pi_{p}(\bar{G})=n-2$.

In both cases, it is determined that $\Pi_{p}(G)+\Pi_{p}(\bar{G}) \leq 2(n-1)$, completing the proof.

\section{Pendant number of graph products}

The rooted product of two graphs $\mathrm{G}_{1}$ and $\mathrm{G}_{2}$, denoted by $\mathrm{G}_{1} \circ \mathrm{G}_{2}$, is the graph obtained by taking $\left|V\left(G_{1}\right)\right|$ copies of $G_{2}$ and identifying one vertex (root) of each copy of $G_{2}$ to the corresponding vertex of $G_{1}$. The following result discusses the pendant number of the rooted products of cycles and paths.

Theorem 25 Let $\mathrm{P}_{\mathrm{n}}$ and $\mathrm{P}_{\mathrm{m}}$ be any two paths and $\mathrm{C}_{\mathrm{n}}$ and $\mathrm{C}_{\mathrm{m}}$ be any two cycles. Then the pendant number of their rooted products are given by;

(i) For $\mathrm{P}_{\mathrm{n}} \circ \mathrm{P}_{\mathrm{m}}$,

$$
\Pi_{p}\left(P_{n} \circ P_{m}\right)= \begin{cases}2(n-1) & \text { if the root vertex is a pendant vertex } \\ 2(n+1) & \text { if the root vertex is an internal vertex. }\end{cases}
$$


(ii) For $\mathrm{C}_{\mathrm{n}} \circ \mathrm{P}_{\mathrm{m}}, \Pi_{\mathrm{p}}\left(\mathrm{C}_{\mathrm{n}} \circ \mathrm{P}_{\mathrm{m}}\right)=2 \mathrm{n}$.

(iii) For $\mathrm{C}_{\mathrm{n}} \circ \mathrm{C}_{\mathrm{m}}, \Pi_{\mathrm{p}}\left(\mathrm{C}_{\mathrm{n}} \circ \mathrm{C}_{\mathrm{m}}\right)=\mathrm{n}$.

\section{Proof.}

(i) Let $u_{1}, u_{2}, \ldots, u_{n}$ be the vertices of $P_{n}$ and $v_{1}, v_{2}, \ldots, v_{m}$ be the vertices of $\mathrm{P}_{\mathrm{m}}$.

Case-1: Let the root vertex of $P_{m}$ be a pendant vertex. Then, in the rooted product $P_{n} \circ P_{m}$, one copy of $P_{m}$ is joined to each of the vertices $u_{1}, u_{2}, \ldots, u_{n}$. The longest path includes the one-point union of $P_{n}$ between two copies of $P_{m}$ (situated at the end points of $P_{n}$ ), yielding 2 pendant vertices. The remaining $n-2$ vertices of $P_{n}$ together with the pendant vertices in the remaining $n-2$ copies of $P_{m}$ result in $2(n-2)$ pendant vertices (see Figure 10 for illustration). Therefore, $\Pi_{p}(G)=2+2(n-2)=2(n-1)$.

Case-2: Let the root vertex of $P_{m}$ be an internal vertex. Then, both end vertices of all the $n$ copies of $P_{m}$ and the end vertices of $P_{n}$ are the end vertices of some paths in the path decomposition of $P_{n} \circ P_{m}$ (see Figure 11 for illustration). Hence, $\Pi_{p}(G)=2(n+1)$.

(ii) For $C_{n} \circ P_{m}$, the collection $V_{p}(G)$ (see 1 ) is constituted by all the vertices of $C_{n}$ together with one end vertex of each of the $n$ copies of the paths $P_{m}$ on the other end as seen in Figure 12. Hence, $\Pi_{p}(G)=2 n$.

(iii) In the rooted product $C_{n} \circ C_{m}$, one copy of $C_{m}$ is joined at each vertex of $C_{n}$. Now take any vertex of degree 2 of $C_{n} \circ C_{m}$ as the first vertex of a path. This path passes through some vertices of the same copy of $C_{m}$, say $C_{m(i)}$, passes through two adjacent vertices of $C_{n}$ and passes through some vertices of the next copy $C_{m(i+1)}$ before it terminates at some vertex of $C_{m(i+1)}$. The next path starts from this end vertex in $C_{m(i+1)}$ and will end at some vertex of the next copy $C_{m(i+2)}$. Continuing like this, one can find out paths which cover all edges of $C_{n} \circ C_{m}$, as shown in Figure 13. Hence, $\Pi_{p}\left(C_{n} \circ C_{m}\right)=n$.

The corona product $\mathrm{G} \odot \mathrm{H}$ of two graphs $\mathrm{G}$ and $\mathrm{H}$ is obtained by taking one copy of $G$ and $|V(G)|$ copies of $H$; and by joining each vertex of the $i^{\text {th }}$ copy of $\mathrm{H}$ to the $i^{\text {th }}$ vertex of $\mathrm{G} ; 1 \leq i \leq|\mathrm{V}(\mathrm{G})|$ (see [8]). When we consider the corona of two paths, for certain initial values of $n$ and $m$, the pendant numbers of $\mathrm{P}_{\mathrm{n}} \odot \mathrm{P}_{\mathrm{m}}$ have already determined (see [3],[6]). They are: 


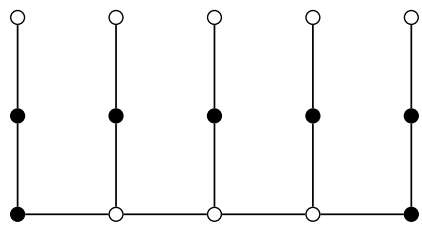

Figure 10: A rooted product $\mathrm{P}_{n} \circ \mathrm{P}_{\mathrm{m}}$ with the root vertex as a pendant vertex.

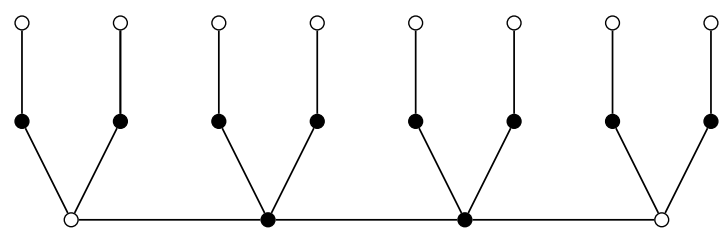

Figure 11: A rooted product $P_{n} \circ P_{m}$ with the root vertex as an internal vertex.

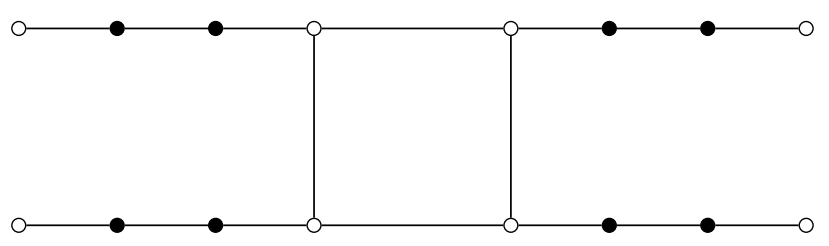

Figure 12: A rooted product $C_{n} \circ P_{m}$.

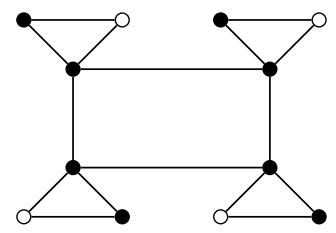

Figure 13: A rooted product $C_{n} \circ C_{m}$.

(i) We can exclude the cases $\mathrm{P}_{1} \odot \mathrm{P}_{1}=\mathrm{K}_{2}, \mathrm{P}_{1} \odot \mathrm{P}_{2}=\mathrm{C}_{3}, \mathrm{P}_{2} \odot \mathrm{P}_{1}=\mathrm{P}_{4}$, whose pendant number is already determined in [3] as 2 .

(ii) Since, $P_{1} \odot P_{m} ; m \geq 3$ is the $n-$ fan graph on $n+2$ vertices, its pendant number is determined in [6] as;

$$
\Pi_{p}\left(P_{1} \odot P_{m}\right)= \begin{cases}m-1 & \text { if } m \text { is odd; } \\ m-2 & \text { if } m \text { is even. }\end{cases}
$$

(iii) $\mathrm{P}_{\mathrm{n}} \odot \mathrm{P}_{1} ; \mathrm{n} \geq 3$ is the comb tree $\mathrm{T}$, whose pendant number is $\Pi_{\mathrm{p}}\left(\mathrm{P}_{\mathrm{n}} \odot \mathrm{P}_{1}\right)=$ $2(n-1)(\operatorname{see}[6])$. 
(iv) $P_{n} \odot P_{2} ; n \geq 2$ is the lever graph $L_{n}$ with pendant number $\Pi_{p}\left(P_{n} \odot P_{2}\right)=$ $n+2$ (see $[6])$.

(v) Since $P_{n} \odot P_{3} ; n \geq 2$ is the diamond neckalce graph $D_{n}$, the pendant number is $\Pi_{p}\left(P_{n} \odot P_{3}\right)=2 n(\sec [6])$.

Theorem 26 The pendant number of the corona product $\mathrm{P}_{\mathrm{n}} \odot \mathrm{P}_{\mathrm{m}}$ with $\mathrm{n} \geq 2$ and $\mathrm{m} \geq 4$ of two paths $\mathrm{P}_{\mathrm{n}}$ and $\mathrm{P}_{\mathrm{m}}$ is given by

$$
\Pi_{p}\left(P_{n} \odot P_{m}\right)= \begin{cases}n(m-1)-2 & \text { if } m \text { is odd } \\ n(m-2)+2 & \text { if } m \text { is even. }\end{cases}
$$

Proof. Let $u_{1}, u_{2}, \ldots, u_{n}$ be the vertices of $P_{n}$ and $v_{1}, v_{2}, \ldots, v_{m}$ be the vertices of $P_{m}$. Let $u_{1}, u_{2}, \ldots, u_{n}$ be the vertices on the root and $v_{11}, v_{12}, \ldots, v_{1 m}$, $v_{21}, v_{22}, \ldots, v_{2 m}, \ldots, v_{n 1}, v_{n 2}, \ldots, v_{n m}$ be the vertices on the crown. Then, the total number of vertices is $n(m+1)$. Let the $j^{\text {th }}$ vertex of the $i^{\text {th }}$ copy of $P_{m}$ be denoted by $v_{i, j}$. The corona product of $P_{n} \odot P_{m}$ where $n \geq 2$ and $m \geq 4$ has two cases:

Case-1: Let $m$ be odd. Since $v_{i 1}, v_{i m} ; 1 \leq i \leq n$ and $u_{1}, u_{n}$ are even degree $(=$ $2 n+2)$ and all other vertices are odd, one can make a path decomposition with the odd degree vertices as the end vertices of every path (see Figure 14 ) and it will be the least (see Theorem 2). Hence, $\Pi_{p}(G)=n(m+1)-$ $(2 n+2)=n(m-1)-2$.

Case-2: Let $m$ be even. Since $v_{i 1}, v_{i m} ; 1 \leq i \leq n$ and $u_{2}, u_{3}, \ldots, u_{n-1}$ are even degree $(=2 n+(n-2))$ and all other vertices are odd, the path decomposition with minimum number of end vertices be the path decomposition with odd degree vertices as the end vertices of every path (see Figure 15). Hence, $\Pi_{p}(G)=n(m+1)-(2 n+n-2)=n(m-2)+2$.

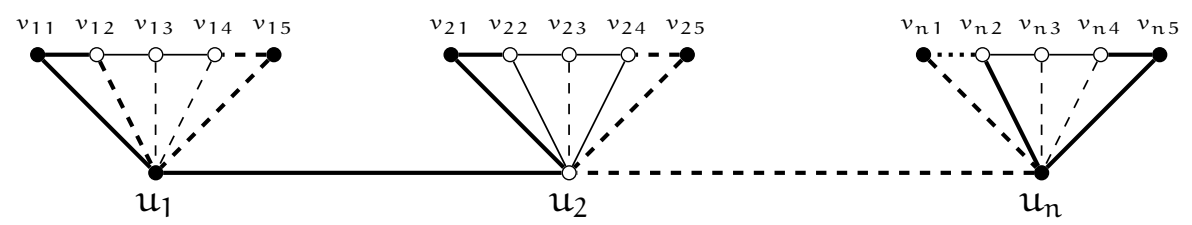

Figure 14: A corona product $\mathrm{P}_{\mathrm{n}} \odot \mathrm{P}_{\mathrm{m}} ; \mathrm{m}$ odd. 


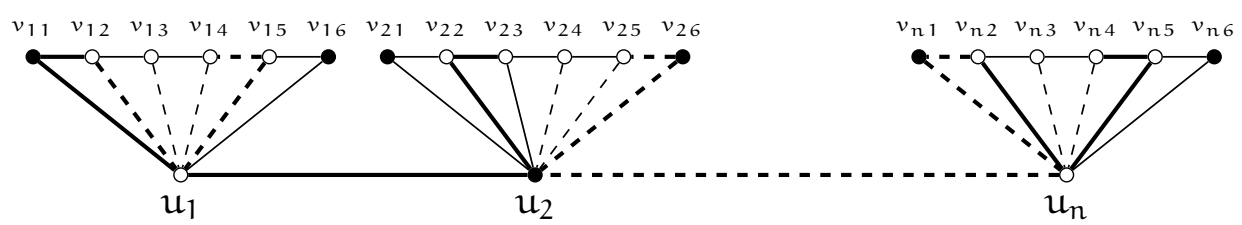

Figure 15: A corona product $\mathrm{P}_{\mathrm{n}} \odot \mathrm{P}_{\mathrm{m}} ; \mathrm{m}$ even.

Theorem 27 The pendant number of the corona product of cycles and paths is given as:

(i) For $\mathrm{C}_{\mathrm{n}} \odot \mathrm{P}_{\mathrm{m}}$,

$$
\Pi_{p}\left(C_{n} \odot P_{m}\right)= \begin{cases}2 n & \text { if } m=1 ; \\ n & \text { if } m=2 ; \\ 2 n\left\lfloor\frac{m-1}{2}\right\rfloor & \text { if } m \neq 1,2 .\end{cases}
$$

(ii) The pendant number of the corona product $\mathrm{C}_{\mathrm{n}} \odot \mathrm{C}_{\mathrm{m}}$ is given by,

$$
\Pi_{p}\left(C_{n} \odot C_{m}\right)= \begin{cases}n m & \text { if } m \text { is even; } \\ n(m+1) & \text { if } m \text { is odd. }\end{cases}
$$

\section{Proof.}

(i) The first two parts of the result follow respectively from Part-(ii) and Part-(iii) of Theorem 25.

Let us now consider the corona product $C_{n} \odot P_{m} ; m \neq 1,2$. Note that for every vertex $v$ of $C_{n}$, the degree of $v$ in $C_{n} \odot P_{m}$ is $m+2$ and for every vertex of each copy of $\mathrm{P}_{\mathrm{m}}$ has degree one more than the degree of the corresponding vertex in $\mathrm{P}_{\mathrm{m}}$. Hence, the following two cases must be considered:

Case-1: Let $m$ be odd. Then, in $C_{n} \odot P_{m}$, every vertex of $C_{n}$ becomes odd degree vertex and all vertices of each copy of $\mathrm{P}_{\mathrm{m}}$, except two (corresponding to the end vertices of $P_{m}$ ) become odd. It is possible to find edge-disjoint paths in $C_{n} \odot P_{m}$ in such a way that the vertices of degree two are not pendant vertices of paths in the path decomposition of $C_{n} \odot P_{m}$ (see Figure 16 for example). Hence, $\Pi_{p}\left(C_{n} \odot P_{m}\right)=n+n(m-2)=n(m-1)$. 
Case-2: Let $m$ be even. Then, in $C_{n} \odot P_{m}$, every vertex of $C_{n}$ remains as an even degree vertex and all vertices of each copy of $P_{m}$, except two (corresponding to the end vertices of $P_{m}$ ) become odd. It is possible to find edge-disjoint paths in $\mathrm{C}_{\mathrm{n}} \odot \mathrm{P}_{\mathrm{m}}$ in such a way that the even degree vertices are not pendant vertices of paths in the path decomposition of $C_{n} \odot P_{m}$ (see Figure 17 for example). Hence, $\Pi_{p}\left(C_{n} \odot P_{m}\right)=n(m-2)$.

Combining the above two cases, it is clear that $\Pi_{p}\left(C_{n} \odot P_{m}\right)=2 n\left\lfloor\frac{m-1}{2}\right\rfloor$.

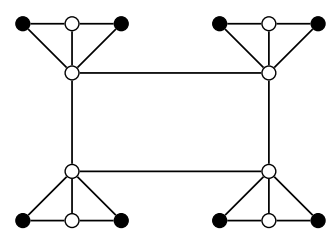

Figure 16: A corona product $\mathrm{C}_{n} \odot \mathrm{P}_{\mathrm{m}} ; \mathrm{m}$ odd.

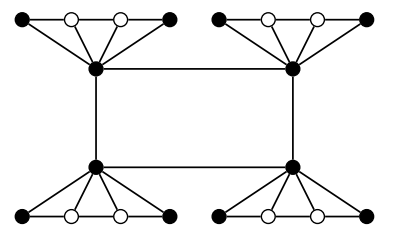

Figure 17: A corona product $\mathrm{C}_{n} \odot \mathrm{P}_{\mathrm{m}} ; \mathrm{m}$ even.

(ii) Let $u_{1}, u_{2}, \ldots, u_{n}$ be the vertices of $C_{n}$ and $v_{1}, v_{2}, \ldots, v_{m}$ be the vertices of $C_{m}$. Let $u_{1}, u_{2}, \ldots, u_{n}$ be the vertices on the root and $v_{11}, v_{12}, \ldots, v_{1 m}$, $v_{21}, v_{22}, \ldots, v_{2 m}, \ldots, v_{n 1}, v_{n 2}, \ldots, v_{n m}$ be the vertices on the crown. In $\mathrm{C}_{\mathrm{n}} \odot \mathrm{C}_{\mathrm{m}}, v_{\mathrm{i} 1}, v_{\mathrm{i} 2}, v_{\mathrm{im}} ; 1 \leq \mathrm{i} \leq \mathrm{n}$ always have odd degree with $\operatorname{deg}\left(v_{\mathrm{ij}}\right)=$ $3 ; 1 \leq j \leq \mathrm{m}$. The total number of vertices in the corona product is $n(m+1)$. There are two possibilities for $C_{n} \odot C_{m}$.

Case-1: Let $m$ be even. Since every vertex of each copy of $C_{m}$ is joined to each vertex of $C_{n}$, the degree of $C_{n}$ remains even, one can make a path decomposition in such a way that none of the vertices of $\mathrm{C}_{\mathrm{n}}$ is the end vertex of any path (see Figure 18) and it will be the least too (see Theorem 2). Thus the pendant number becomes $n(m+1)-n=n m$. 


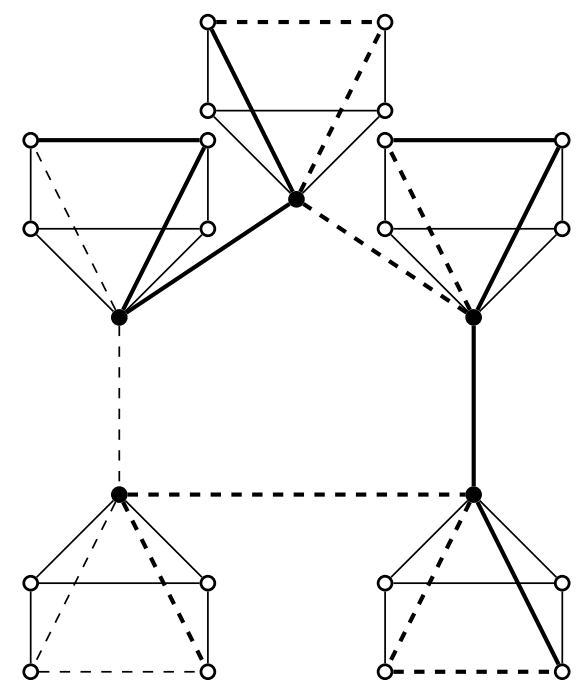

Figure 18: A corona product $C_{n} \odot C_{m} ; m$ even.

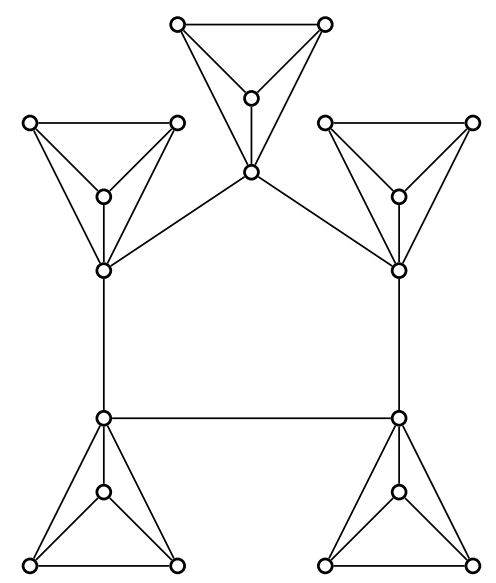

Figure 19: A corona product $\mathrm{C}_{n} \odot \mathrm{C}_{\mathrm{m}} ; \mathrm{m}$ odd.

Case-2: Let $m$ be odd. Then, all the vertices of $C_{n} \odot C_{m}$ become odd. Thus, by Theorem 2, pendant number is $\mathfrak{n}(\mathrm{m}+1)$ (see Figure 19), the entire vertex set of the resultant graph. 


\section{Conclusion}

In this paper, we discussed certain properties of the pendant number of a given graph $\mathrm{G}$. We have determined the pendant number of corona products and rooted products of paths and cycles. We have also obtained some bounds for the pendant number for some specific derived graphs. Corresponding to any natural number $n$, the existence of a connected graph with pendant number $n$ has also been established in this study. These studies can further be extended to other general graph classes, graph products and other graph operations. The relation of pendant numbers with other popular parameters like domination number, covering number, chromatic number etc. can also be studied.

\section{References}

[1] D. B. West, Introduction to Graph Theory, Prentice Hall of India, New Delhi, 2005. $\Rightarrow 24$

[2] F. Harary, Graph Theory, Narosa Publishing House, New Delhi, 2001. $\Rightarrow$ 24

[3] J. K. Sebastian, J. V. Kureethara, Pendant number of graphs, Int. J. Appl. Math., IJAM 31, 5 (2018) 679-689. $\Rightarrow 25,34,35$

[4] J. K. Sebastian, J. V. Kureethara, N. K. Sudev, C. Dominic, On star decomposition and star number of some graph classes, Int. J. Scien. Res. Mathe. Stati. Sci., IJSRMSS, 5, 6 (2018) 81-85. $\Rightarrow 25$

[5] J. K. Sebastian, J. V. Kureethara, N. K. Sudev, C. Dominic, On the pendant number of some new graph classes, Res. Rev. Discrete Math. Structures RRDMS, 6, 1 (2019), 15-21. $\Rightarrow 34,35,36$

[6] J. K. Sebastian, J. V. Kureethara, N. K. Sudev, The pendant number of line graphs and total graphs, communicated. $\Rightarrow 34,35,36$

[7] N. K. Sudev, K. A. Germina, A study on topological integer additive set-labeling of graphs, Electron. J. of Graph Theory Appl. Electronic Journal, 3, 1 (2015), 70-84. $\Rightarrow 29$

[8] Y. N. Yeh, I. Gutman, On the sum of all distances in composite graphs, Discrete Math. Discrete, 135, 1-3 (1994), 359-365. $\Rightarrow 34$

[9] X. Zhang, H. Zhang, Some graphs determined by their spectra, Lin. Alg. and Its Appl. Linear, 431, 9 (2009), 1443-1454. $\Rightarrow 32$ 